\title{
El Currículo en la Práctica y su Relación con las Tecnologías del Aprendizaje y el Conocimiento
}

\author{
The Curriculum in Practice and its Relationship with Learning and Knowledge \\ Technologies
}

Marcela Zeballos ${ }^{1}$ \section{RTED
- EDICIÓN: Nesiliencia
aradigmàtica}

Recibido: 6/julio/2021

Aceptado: 6/octubre/2021

Publicado: 29/noviembre/2021

Páginas: 117-129

\&aís

${ }^{1}$ Argentina

\section{IIIII Institución \\ ${ }^{1}$ Instituto Superior de Formación Docente}

\section{Correo Eletrónico}

1marcelazeballos3@gmail.com

\section{(D) ORCID}

${ }^{1}$ https://orcid.org/0000-0002-8613-5998

\section{Citar así: CfAPA / IEEE}

Zeballos, M. (2021). El Currículo en la Práctica y su Relación con las Tecnologías del Aprendizaje y el Conocimiento . Revista Tecnológica-Educativa Docentes 2.0, 1(1), $117-129$.

https://doi.org/10.37843/rted.v1i1.261

M. Zeballos, "El Currículo en la Práctica y su Relación con las Tecnologías del Aprendizaje y el Conocimiento ", RTED, vol. 1, n. ${ }^{\circ}$, pp. 117-129, nov. 2021.

\section{Resumen}

La sociedad actual está signada por nuevas ecologías cognitivas. En este sentido es fundamental sentar las bases para analizar y comprender el oficio de enseñar en el marco de estos nuevos espacios. El presente estudio tuvo como objetivo analizar un caso práctico sobre el uso pedagógico de las tecnologías en el nivel Superior. Se utilizó el método cualitativo para el análisis de los diferentes datos recolectados A partir del estudio de caso se accedió a múltiples fuentes de información permitiendo corroborar los resultados obtenidos otorgándole mayor confiabilidad a los hallazgos. Los resultados arrojaron una serie de posibilidades y debilidades al implementar las tecnologías, evidenciaron distintas lecturas e interpretaciones por parte de los docentes al abordar los documentos curriculares. Finalmente, los estudiantes se sintieron más involucrados al trabajar de manera autónoma en su propio ritmo de aprendizaje transitando hacia un cambio de paradigma cultural. El estudio permitió deducir la reconstrucción de concepciones de parte del docente dirigidas hacia un enfoque integral de aprendizaje. Diferentes maneras de lectura de materiales curriculares evidenciaron múltiples interpretaciones a partir de biografías profesionales, contextos de desempeño y circunstancias sociales. Por otra parte, fue fundamental tender hacia un currículo flexible, es decir organizando la enseñanza desde la diversidad, con enfoques atentos a diferentes ritmos de aprendizaje.

Palabras clave: Currículo, tecnología del aprendizaje y del conocimiento, práctica docente.

\section{Abstract}

New cognitive ecologies mark today's society. In this sense, it is essential to lay the foundations to analyze and understand the profession of teaching within the framework of these new spaces. The present study aimed to analyze a practical case on the pedagogical use of technologies at the Higher level. The qualitative method was used for the analysis of the different data collected. From the case study, multiple sources of information were accessed, allowing the results to be corroborated, granting more excellent reliability to the findings. The results showed a series of possibilities and weaknesses when implementing the technologies; they showed different readings and interpretations by the teachers when addressing the curricular documents. Finally, the students felt more involved by working autonomously at their own pace of learning, moving towards a change in the cultural paradigm. The study allowed to deduce the reconstruction of conceptions from the teacherdirected towards a comprehensive approach to learning. Different ways of reading curricular materials evidenced multiple interpretations based on professional biographies, performance contexts, and social circumstances. On the other hand, it will be essential to tend towards a flexible curriculum, organizing teaching from diversity, with conservative approaches to different learning rates.

Keywords: Curriculum, learning and knowledge technology,teaching practice. 


\section{Introducción}

La sociedad actual está signada por nuevas ecologías cognitivas. En este sentido es fundamental sentar las bases para analizar y comprender el oficio de enseñar en el marco de estos nuevos espacios que configuran escenarios educativos a partir del reconocimiento de prácticas docentes hibridadas por modalidades virtuales y presenciales (Piscitelli, 2011). En este contexto, el oficio de enseñar se encuentra impactado por las tecnologías; asimismo, se encuentran situaciones de confluencia de escenarios y mezcla de modelos. Estamos en presencia en un nuevo escenario de aprendizaje como entidades dinámicas caracterizadas por un conjunto de contextos formales, no formales $\mathrm{y} / \mathrm{o}$ informales que brindan oportunidades para el aprendizaje. (Barron,2006). Los espacios y tiempos flexibles potencian diferentes estilos de aprendizaje necesitando cambios de actitudes, competencias y roles de parte de docentes y estudiantes.

De acuerdo con, Prensky (2010), las nuevas generaciones de estudiantes utilizan ordenadores, videojuegos, teléfonos celulares entre otras herramientas digitales. La enseñanza debe diseñarse a partir de la cultura juvenil, incrementando la motivación, evadiendo la evasión escolar, y el diseño de nuevos currículos. (Marchesi 2009). Una nueva ecología de aprendizaje requiere de un cambio de paradigma pedagógico, en donde es fundamental trabajar con trayectorias educativas personalizadas como camino hacia una sociedad del conocimiento.

El currículo cobra vida en la escuela materializándose en las experiencias cotidianas la que se encuentra fuertemente surcada por tradiciones institucionales, historias y matrices de formación docente, contextos y tramas de sentido (Rockwell 1986). En el nuevo paradigma el currículo se reposiciona hacia un aprendizaje integral e inclusivo que resulta necesario para funcionar eficazmente en el siglo XXI. Por las razones antes expuestas, las instituciones educativas tienen que reinventarse y repensar una nueva relación pedagógica, tratando de articular estrategias, recursos y prácticas de enseñanza para convertir espacios democráticos de conocimiento. Es decir, un aprendizaje situado, como metodología docente basada principalmente en situaciones cotidianas y en la solución de problemas.

El objetivo del trabajo apuntó a comprender y profundizar un caso práctico sobre la utilización de las herramientas tecnológicas y su relación con el currículo en la práctica del Nivel Superior en el espacio curricular Didáctica y Currículo del Nivel primario. Las preguntas que motivaron la realización de dicha investigación fueron las siguientes: ¿Cómo se puede abordar el currículo escolar en la nueva ecología del aprendizaje? ¿Cuáles son los aprendizajes imprescindibles y deseables en el nivel superior? ¿Qué rol juegan las tecnologías en la personalización de los aprendizajes?, ¿Qué estrategias tecnológicas se utilizaron para el trabajo de la interculturalidad?

\section{Metodología}

El diseño metodológico fue el estudio de caso desarrollado a partir de la metodología cualitativa. Se requirió de un plan para la selección del escenario y la recopilación de los datos. Fue un diseño emergente circular, cada decisión dependió de la información previa. La selección de la muestra fue un caso único y los criterios que sirvieron como guía para la selección de la misma fueron los siguientes:

1. Diseño de un proyecto de enseñanza y aprendizaje en el Nivel Superior

2. Cátedra Didáctica y Currículo del Nivel primario

3. Segundo año de la Formación Docente

El estudio se desarrolló en el marco de la clase Didáctica y Currículo del Nivel Primario correspondiente al segundo año de Formación Docente. El destinatario estuvo conformado por 15 estudiantes, de género femenino y cuyo rango de edad oscilaba entre 23 y 35 años, perteneciente a una institución de gestión pública de la provincia de Buenos 
Aires. La asignatura tiene como objetivo vivenciar y comprender la realidad y práctica educativa, reflexionando sobre la propia praxis como maestros en formación a fin de enriquecer su desarrollo profesional.

La recopilación de los datos y su respectivo análisis fueron procesos interactivos, Las siguientes fases de investigación explicadas a continuación, demuestran los procesos para la selección de la muestra, el análisis de datos y su respectiva interpretación.

- Fase 1 planificación: para el planteo del presente se presentaron las preguntas de investigación, que orientaron la recolección de datos.

- Fase 2: inicio de la recolección de datos: se organizó y se reunió los datos recopilados para su análisis formal.

- Fase 3 recopilación de datos básicos: se comenzó a oír, ver y leer lo que estaba sucediendo. Durante esta fase se siguió seleccionando estrategias para la recolección de datos y de los informantes.

- Fase 4 finalización: esta fase dio lugar al análisis formal por descubrimiento de los datos y a la elaboración de formas de presentar la información.

La principal herramienta para el análisis de los datos fue la contrastación para identificar los segmentos de datos, dar un título al tema, categorías y clasificarlos. Las estrategias de descubrimiento fueron empleadas para desarrollar ideas tentativas durante la formulación de datos.

Algunas de las estrategias que se han empleado fueron:

1. Escritura en el diario de bitácoras para separar los datos descriptivos de los comentarios e interpretaciones del investigador.

2. Escritura de resúmenes de los análisis y de las entrevistas para centrar el tema. Al realizar resúmenes el investigador seleccionó aspectos relevantes y a reorganizar la información con criterios lógicos.

3. Se definió diversos temas para el desarrollo de categorías y conceptos.

4. Se buscó modelos que se repetían y que pudieran convertirse en nuevos temas

5. Se examinó la bibliografía para analizar el apoyo o la discrepancia con las observaciones.

La triangulación en investigaciones cualitativas aparece como posibilidad a fin de que el investigador tenga la posibilidad de encontrar distintos caminos para la comprensión del fenómeno. Se utilizó la triangulación de instrumentos de recolección de datos como la observación, la entrevista y el análisis de documentos. Dicha estrategia se utilizó para contrastar distintos instrumentos de recolección de datos y el análisis del material de forma cualitativamente. La selección de artefactos fue una estrategia para obtener datos cualitativos que requirió de trabajo de análisis creativo para poder conseguir datos relevantes. Entre los documentos oficiales analizados podemos destacar, el Diseño Curricular del Nivel Superior y las planificaciones áulicas. El análisis y la selección de artefactos requirió de las siguientes estrategias: localización de artefactos, identificación, análisis, critica e interpretación. Se comenzó con la recolección de los primeros datos, segmentando unidades significativas, acontecimientos o escenas esenciales. Todo este proceso requirió de una visión integral, lo que facilitó la interpretación de los datos más pequeños.

El paso de un entorno presencial a otro virtual produjo cierta tensión en los docentes, por ausencia de formación pedagógica didáctica en el uso tecnología. En algunos casos los profesores solían trabajar con estrategias presenciales tradicionales en aulas virtuales, desconociendo la especificidad de dicha modalidad. Será fundamental coordinar estas estrategias para construir un modelo integral.

\section{Resistencia de los Estudiantes al Cambio de Modelo}


Fuente de Información Profesora de la Catedra Didáctica y Currículo del Nivel Primario. Al aplicar una nueva metodología activa se observó la resistencia al cambio por parte de los estudiantes. El aprendizaje activo es aquel compuesto por los siguientes ingredientes: motivación, implicación, trabajo constante y atención. Por tal motivo se llevó a cabo distintas actividades para desarrollar el pensamiento autónomo familiarizando a los estudiantes con una nueva modalidad de aprendizaje a partir del cambio en actitudes confeccionando un diario de clase. A continuación, se presentan las estrategias reflexivas (ver Figura 1) solicitadas a los estudiantes:

\section{Figura 1}

Estrategias Reflexivas ante el Cambio de Modelo de Aprendizaje

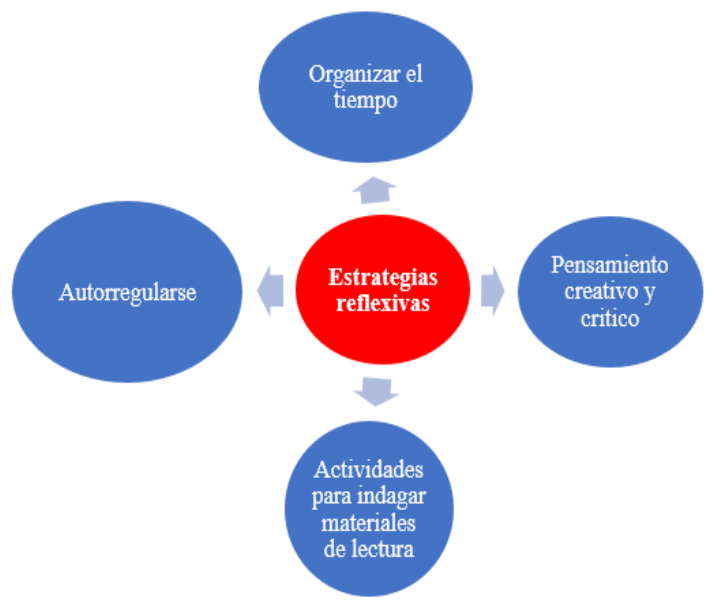

Nota. Según el análisis de las entrevistas a la docente de la cátedra se extraen las estrategias reflexivas para trabajar junto a los estudiantes, elaboración propia (2021).

Las estrategias reflexivas no solo permiten al estudiante comprender los conocimientos teóricos desde una perspectiva problematizadora sino también para el cambio en el modelo de aprendizaje. En este sentido, en el nuevo modelo, el foco debe estar puesto en el estudiante y en su desarrollo integral. Será preciso conocer el perfil del egresado y las expectativas del estudiante para poder trabajar con las resistencias al cambio. $\mathrm{Si}$ embrago las tecnologías, permitieron desarrollar nuevos espacios y los docentes tuvieron la posibilidad de construir nuevas culturas con creencias y valores que consideraban pertinentes para la sociedad actual.

\section{Planificación Didáctica}

Fuente de Información Planificación Didáctica de la Disciplina. Reflexionar sobre el cambio de paradigma nos obliga a repensar el diseño de planificaciones de clase. El modelo Flipped classroom, constituyó una buena alternativa para el desarrollo del trabajo colaborativo, el pensamiento autónomo otorgando protagonismo en los estudiantes. Padlet permitió crear murales colaborativos para la realización de un diagnóstico de trayectorias educativas en los estudiantes colaborativos. A partir del diseño de planificaciones didácticas se tuvo en cuenta (ver Figura 2):

\section{Figura 2}

\section{Aspectos en la Planificación Didáctica}

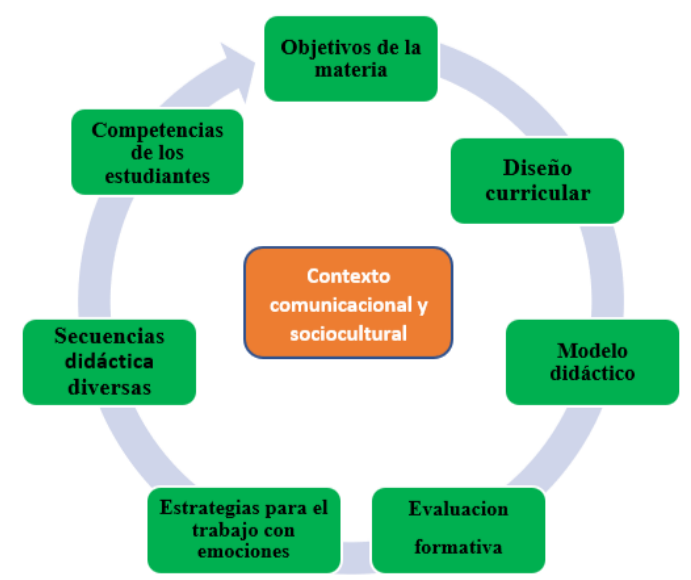

Nota. De acuerdo con el análisis de la planificación didáctica de la materia Didáctica y Currículo se observó una unidad didáctica integrada promoviendo aprendizajes de habilidades conceptuales, procedimientos y actitudes para el desarrollo de destrezas que permitieran establecer nuevas relaciones conceptuales, elaboración propia (2021).

En la sociedad del conocimiento los medios de comunicación, las tecnologías y los servicios en línea pasan a ocupar un papel importante en la educación. Este hecho conlleva que en el campo de la Didáctica se reconozcan nuevos conceptos que revelan confrontaciones en el campo de la enseñanza tradicional. En el diseño de planificaciones 
didácticas se tuvo en cuenta el rol del estudiante frente a su aprendizaje, fue fundamental recabar información relevante y de esta manera el docente puedo contextualizar su intervención didáctica al tener en cuenta las necesidades del educando.

\section{Resistencia del Docente al Cambio}

\section{Fuente de Información Entrevista a} Docentes. Tanto para el estudiante como para los docentes, el traslado de un entorno presencial a uno virtual supuso adaptación y aprendizaje. Este proceso llevó un tiempo para trabajar la resistencia al cambio. Los saberes del docente se encontraban tensionados por los cambios culturales entre los que se encuentran los medios tecnológicos. Entre las resistencias que se presentaron en el proceso de enseñanza y aprendizaje virtual fueron:

- Tecnológicas: conocimiento limitado de la tecnología por parte de estudiantes y docentes.

- Culturales: profesores adaptados a la presencialidad no acostumbrados a gestionar su propio conocimiento.

- Didácticas: Ausencia de diseño para planificar los contenidos en aulas virtuales y con poca formación en enseñanza a distancia.

El docente de la catedra expresa:

"Dar clase a través del aula virtual me dio sensación de soledad y de aislamiento por no mantener diálogo con mis colegas"

"Prefiero lo familiar al extraño o lo nuevo, es decir, actuar según un modelo ya adquirido me da sentimientos de confianza y seguridad"

"Cuando me recibí como profesora aprendi de una manera y esta manera suele persistir. Realizo cursos de capacitación y regreso al aula vuelvo al sistema anterior ya que el contexto no ha cambiado"

Para el autor Fernández, (1992) la intervención destinada a eliminar o reducir la resistencia al cambio debe estar basada en la colaboración, en la acción conjunta y la autonomía institucional. A partir del desarrollo profesional docente, los desafíos deben apuntar no solamente en la manera que aprende el estudiante, sino también en el contexto en el que se relaciona. Los docentes solamente estarán comprometidos (ver Figura 3) con el cambio si han tenido oportunidad en tomar decisiones sobre los mismos.

Figura 3

La Gestión en el Aula Virtual
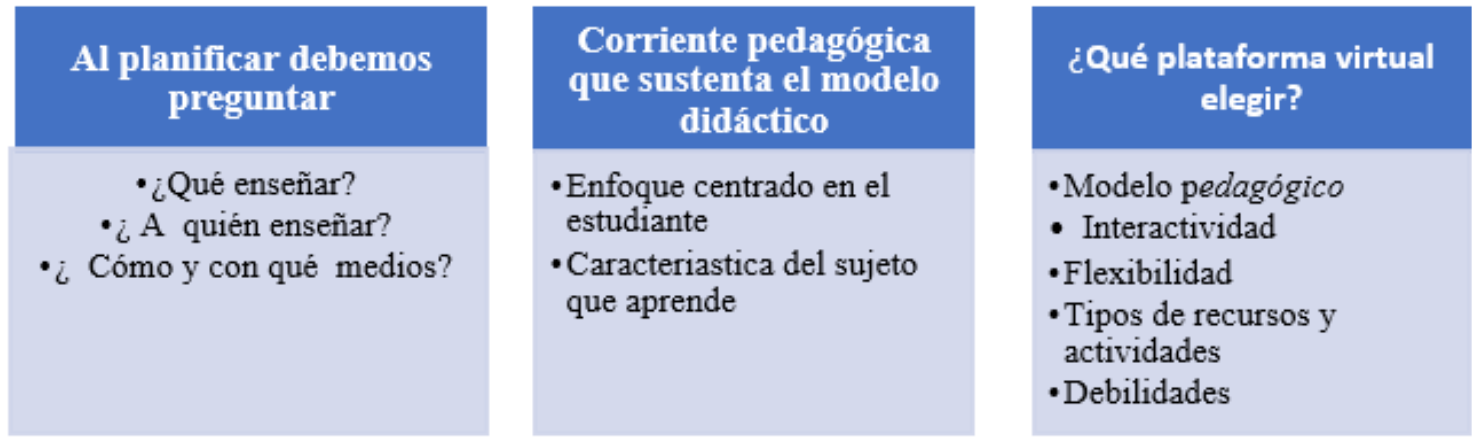

Nota. Elementos previos para tener en cuenta en la gestión del aula virtual, elaboración propia (2021).

El rol del docente deberá ser un especialista en su disciplina, guiar, acompañar, evaluar y estimular al estudiante en su proceso de aprendizaje. Es importante a la hora de gestionar el aula virtual tener en cuenta las características del estudiante y su cultura.

Tanto la educación presencial como virtual va más allá de la presentación de contenidos, ya que los estudiantes deberán construir ideas y 
pensamientos a partir de sus saberes previos. Además, de la relación entre contenidos, estrategias y actividades debe enfocarse en provocar motivación para mantener el interés constante.

\section{Configuración de Ambientes Virtuales de Aprendizaje}

Fuente de Información: Planificación

Didáctica. Un ambiente virtual de aprendizaje es el espacio que se crea para propiciar el intercambio de conocimientos entre docentes y estudiantes, a partir de plataformas virtuales que favorecen la comunicación de ideas e interacciones durante el proceso de aprendizaje. En este espacio virtual se puede tener acceso en cualquier momento a contenidos, recursos, actividades facilitando el proceso de aprender. Al realizar un análisis de la planificación didáctica se observó la importancia del aprendizaje intercultural a partir de recursos, acceso a la información intercambios y reflexiones colectivas. El apoyo de las tecnologías generó la capacidad de incrementar la comunicación, elaboración conjunta de información, diálogo y aprendizaje colaborativo. De esta manera los estudiantes adquirieron distintas capacidades para reflexionar (ver Figura 4) sobre su propia cultura y la de los demás.

\section{Figura 4}

Estrategias y Recursos Tecnológicos Atentos a la Interculturalidad

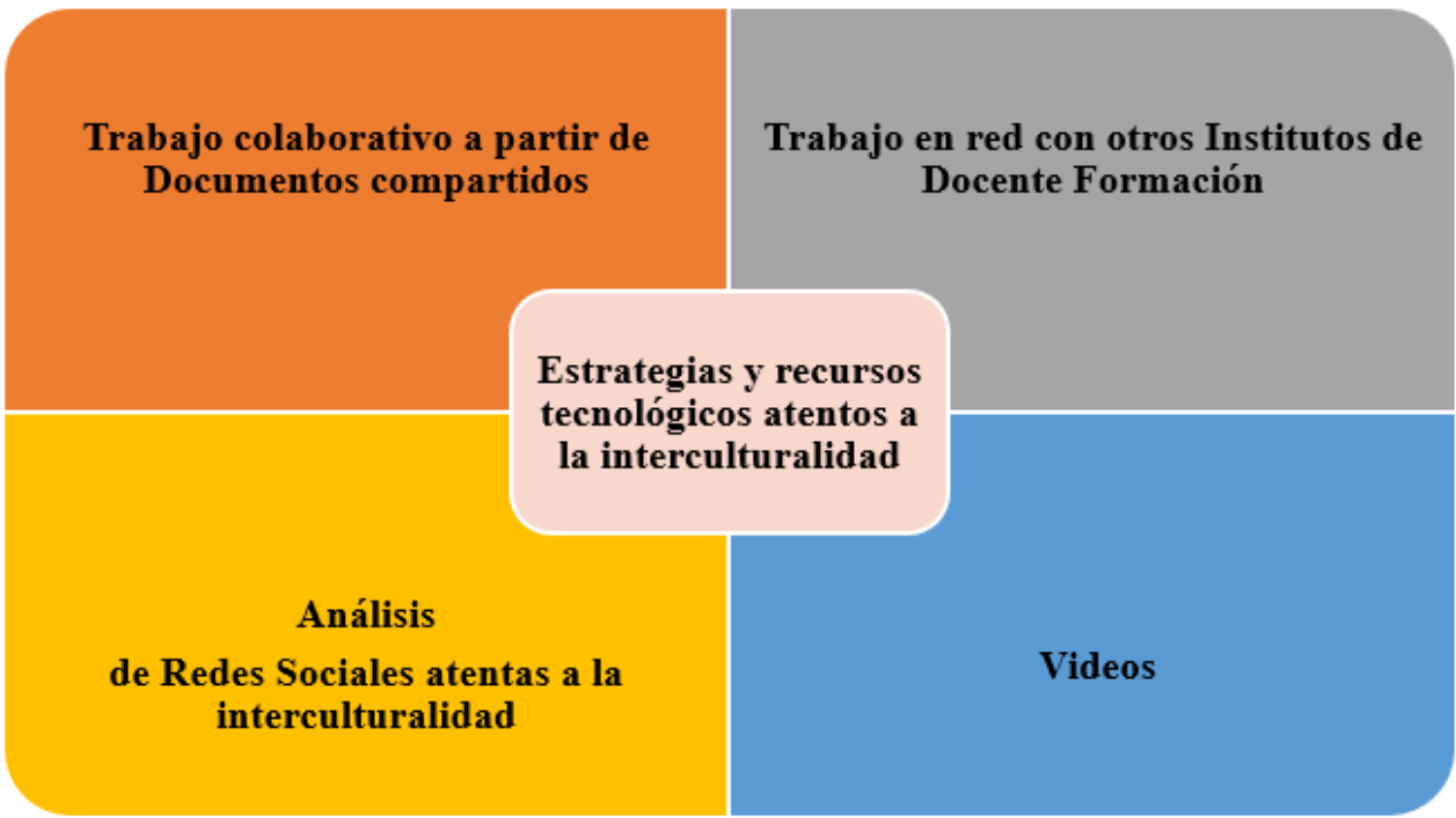

Nota. De acuerdo con el análisis de la planificación didáctica se observó diferentes recursos para el trabajo de la interculturalidad, elaboración propia (2021).

\section{Utilización de Redes Sociales}

Fuente de Información: Planificación Didáctica. Las redes sociales se convirtieron en un recurso didáctico que permitió el aprendizaje colaborativo e involucraron espacios de diálogo, e incluso ofrecieron un gran potencial como recurso para el abordaje de metodologías activas. Plataformas como
Facebook, WhatsApp. Meet y Zoom (ver Figura 5) permitieron a los estudiantes desarrollar capacidades digitales y trabajar de manera colaborativa. De esta manera, el docente no permaneció en su rol pasivo como transmisor de conocimientos, sino que pudo fomentar la cooperación entre los estudiantes. 
El currículo en la práctica y su relación con las Tecnologías del Aprendizaje y el Conocimiento.

\section{Figura 5}

Redes Sociales y Herramientas Utilizadas Durante el Proceso de Enseñanza-Aprendizaje

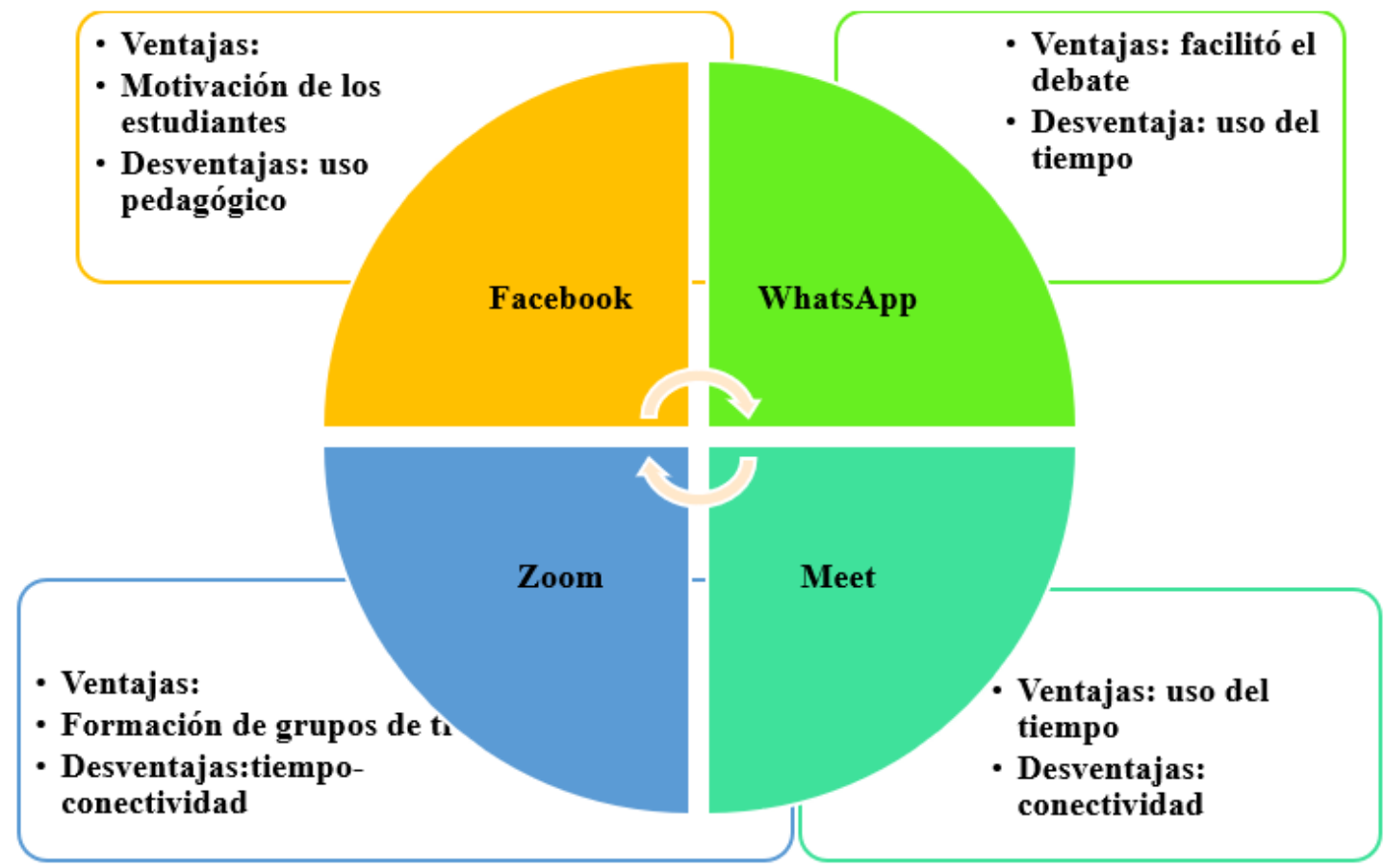

Nota. De acuerdo con el análisis de la planificación didáctica se pudo observar la importancia de la utilización de herramientas tecnológicas para el desarrollo de competencias cognitivas y emocionales, elaboración propia (2021).

Las redes sociales proporcionan un amplio acceso a la información y a los recursos de información, reducen las barreras a la interacción de grupo y a la comunicación (Valenzuela, 2013; Muñoz, Fragueiro \& Ayuso, 2016). Algunas propuestas para sacar el máximo provecho a estas herramientas pueden ser la generación de debates y estrategias para motivar a los estudiantes fomentando la participación y la creatividad. La utilización de plataformas como Zoom y Meet ayudaron al docente a potenciar la comunicación con los estudiantes. Es preciso destacar que lo pedagógico debe primar sobre lo tecnológico.

La profesora de la cátedra expresa:

Adaptarse al nuevo sistema no fue fácil ya que hice muy pocos cursos relacionados con enseñar a distancia. Los problemas más comunes fueron: la conexión muy débil, interferencias en el audio o la incapacidad de compartir presentaciones. Lo que no se hacer todavía es integrar las prácticas de campo a la enseñanza virtual.

Según el Diseño Curricular para la Formación Docente la materia Didáctica y Currículo del Nivel Primario, expresa:

La génesis de ambos objetos de estudio muestra escenarios $e$ intencionalidades que responden a tradiciones diversas. Confluyen en el estudio del vínculo entre sujetos y objetos de conocimiento que se construyen en el propio proceso relacional, configurado sociocultural $e$ históricamente y orientado por los fines de la enseñanza. En esta materia se pretende fundamentar una construcción curricular colectiva, que permita identificar y orientar la particularidad de las prácticas de la enseñanza. 
Existen diversidad de posturas teóricas y prácticas acerca de la Didáctica. Una disciplina de naturaleza pedagógica que necesita apoyarse en teorías psicológicas de aprendizaje para acercarse a situaciones donde se produce el aprendizaje. La didáctica comunicativa da mucha importancia a la relación de los grupos. Es decir, que el campo de la didáctica es comprendido como el conjunto de sistemas sometidos a interacciones específicas que permitan y favorezca el acercamiento, la comunicación, la puesta en marcha de organizaciones en las que cada vez se alcancen cuotas de conciencia y se elaboren modos de acción y participación (Popp, W,1976). La nueva realidad ha generado nuevas prácticas en la implementación del currículum (Gimeno Sacristán, 1998), modificando la intervención docente generando nuevos desafíos al planificar en un aula virtual.

\section{Estrategias para el Desarrollo de Trayectorias Educativas}

Fuente de Información Entrevista a la Profesora de la Cátedra. El seguimiento de los estudiantes en contextos virtuales implicó diseñar tareas con diferentes herramientas didácticas. El seguimiento de dichas estrategias permitió:

1. Análisis de progreso ajustando a la planificación didáctica.

2. Revisar actividades y recursos didácticos.

3. Modificación de estrategias pedagógicas.

4. Retroalimentación de la evolución del aprendizaje.

5. Autoevaluación de la práctica docente.

A continuación, se socializan las expresiones narrativas por parte de la profesora de cátedra:

Se ofrecieron estrategias Didácticas para que los estudiantes pudieran reflexionar criticamente sobre su trayectoria. Esta tarea se llevó a cabo a partir de mecanismos de retroalimentación, en los momentos virtuales. Por otra parte, los encuentros en la plataforma Google Meet y Zoom estuvieron centrados en el estudiante, pero sin perder de vista los contenidos. Creo que el contenido fue muy importante, pero sin vinculo no hay aprendizaje. Utilicé estrategias para modular la voz, tener una postura relajada, energía positiva y responder a preguntas de manera amigable. Algunos estudiantes no contaban con dispositivos tecnológicos y/ conexión a internet, $y$ ante esta situación prepare guías de aprendizaje.

Las emociones juegan un rol fundamental en la creación de experiencias de aprendizaje.

Otro tema que quiero compartir es el abandono de los estudiantes en las clases virtuales por no poseer internet y no poseer habilidades tecnológicas.

Durante la entrevista a la profesora se observa el seguimiento pedagógico en distintas oportunidades para demostrar lo aprendido por los estudiantes. Se utilizaron distintos recursos para sostener la comunicación. Si bien algunos estudiantes no contaban con dispositivos tecnológicos y/o conexión se organizaron otras actividades en relación con el desarrollo de habilidades. Para el cuidado de la trayectoria en los estudiantes del Nivel Superior será fundamental tener en cuenta los aprendizajes flexibles y creativos, a partir de la virtualidad o en un formato físico.

\section{Estrategias para el Acompañamiento en la Lectura de Textos Académicos en el Nivel Superior}

\section{Fuente de Información Entrevista a} la Profesora de la Materia. Durante el proceso de aprendizaje se encontraron algunos obstáculos relacionados con los textos académicos utilizados en la materia. Los estudiantes tuvieron dificultades de seleccionar y organizar la información La búsqueda buscaban información se realizan por internet, pero sin criterios de búsqueda. 
Deben ser adoptadas nuevas estrategias (ver Figura 6) que aporten innovación a la enseñanza, que son directamente responsables por la diseminación del conocimiento, en un ambiente diversificado y complejo que es el aula virtual (Sánchez, 2019).

A continuación, se socializa las expresiones de la profesora de la materia:

Una buena manera de generar aprendizajes autónomos fue a través de la aplicación de porfolios para registrar y reflexionar lo aprendido. A partir de esta estrategia se diseñaron actividades de lectura y comprensión de textos. Una vez cumplida esa tarea los estudiantes, realizaron actividades de retroalimentación accediendo a su porfolio a través del sitio web Symbaloo. Se subieron tareas de manera organizada. Esta herramienta tecnológica permitió integrar los contenidos y evaluar su aprendizaje.

\section{Figura 6}

Estrategias para la Comprensión de Textos Académicos en la Virtualidad

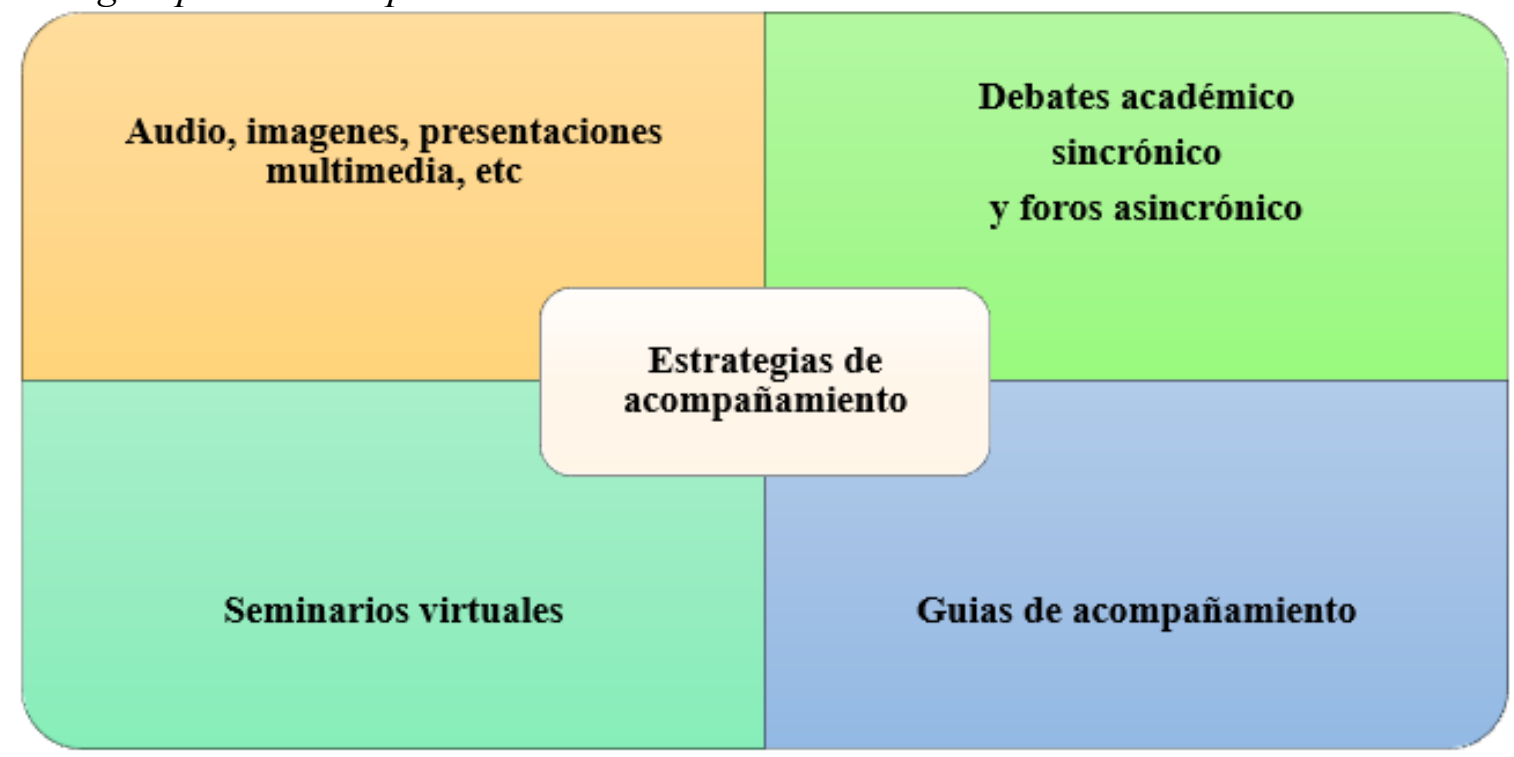

Nota. De acuerdo con el análisis de la planificación didáctica permite reconocer las herramientas para mejorar la comprensión de la lectura de textos argumentativos en el nivel superior, elaboración propia (2021).

Una de las problemáticas que se observa con frecuencia en el Nivel Superior fue la falta de comprensión de textos académicos, por lo que es necesario facilitar estrategias que mejoren esta competencia. El pasaje de la hetero a la autorregulación se irá produciendo en la medida en que los alumnos puedan ir apropiándose de instrumentos para la comprensión lectora que podamos brindarles como docentes (Coll, 1985). Entonces, ¿Cómo favorecer que esto ocurra en contextos virtuales? En este sentido, el acompañamiento pedagógico del docente es fundamental en el uso efectivo de la plataforma. Los resultados del estudio es la relevancia del rol del docente en el uso efectivo de la plataforma. A pesar de las estrategias de acompañamiento que se implementan para la comprensión lectora en los estudiantes requieren además del seguimiento continuo del docente. $\mathrm{La}$ tecnología cuando es utilizada de manera adecuada permite que los estudiantes logren ser autosuficientes y adquieran habilidades que mejoren su desempeño profesional (Mujica-Sequera, 2020).

\section{Retroalimentación de Aprendizajes en Espacios Virtuales}

La retroalimentación o feedback es entendida como cualquier información, que se proporciona a quien desempeña una acción cualquiera, respecto de ese desempeño (Ross 
y Tronson, 2005), por lo que se podría considerar que tanto los aciertos, errores, fortalezas y debilidades de los estudiantes es muy importante para el logro de aprendizajes significativos.

Se comparte las expresiones de la profesora de la cátedra:

La utilización de tutoría como estrategia fue utilizada para detectar dificultades potenciando diversas habilidades en los estudiantes. Por otra parte, se orientó en la construcción en la autonomía del aprendizaje, gestión de las emociones, actitud crítica, y promoción del diálogo en espacios virtuales.

Fue muy valioso las actividades prácticas con ayuda de textos, apuntes apoyados con recursos tecnológicos. También se seleccionaron, evaluaron, $y$ organizaron información a partir de distintos criterios, para su socialización.

Otro aspecto fundamental fue el uso de glosarios para memorizar en forma comprensiva y explicar los diversos temas. Por otro lado, se realizaron ejercicios aplicando glosarios estableciendo definiciones a través del recurso didáctico Padlet socializando por medio de diferentes soportes, blog, porfolio o cuaderno de bitácoras.

La retroalimentación entre docentes y estudiantes es fundamental para promover la motivación. A veces, cuando no se cuenta con estrategias adecuadas $y$ un buen acompañamiento de parte del docente, los estudiantes pueden percibir a la retroalimentación como instancia negativa.

La devolución sobre el desempeño debe basarse en la tarea que se está haciendo y aquello que se está realizando de manera incorrecta, incluirse con un modo amable para que el estudiante crea que puede realizar las correcciones. Las tutorías son fundamentales para que los estudiantes tengan la oportunidad recibir atención personalizada. Por otra parte, el glosario virtual, como otro recurso para la retroalimentación, se convirtió en un elemento valioso, para conceptualizar y como espacio para la construcción colaborativa junto al estudiante. Para asimilar e internalizar el conocimiento fue necesario motivar, acompañar, utilizar debates, intercambios y el establecimiento de vínculos (ver Figura 7).

Un estudiante expresa:

La herramienta de gran utilidad fueron los foros, porque dieron posibilidad de reflexión crítica muy atractivo para practicar, revisar conceptos $y$ autocorregirse.

\section{Figura 7}

Herramientas que se Utilizaron para la Retroalimentación

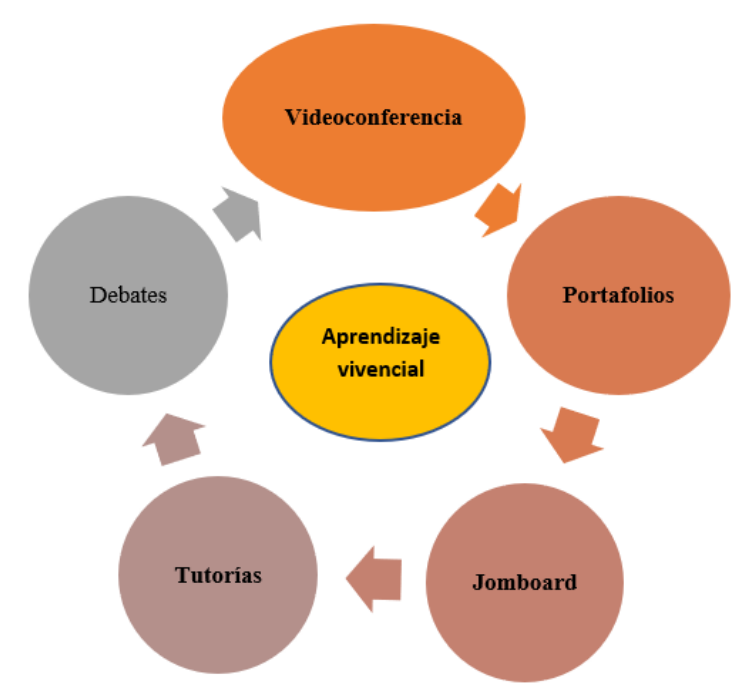

Nota. De acuerdo con la profesora entrevistada se Eligio cada herramienta de retroalimentación para considerar las diversas evidencias de los aprendizajes, elaboración propia (2021).

Para que la evaluación se pueda constituir en una potente herramienta para el proceso de enseñanza y aprendizaje es imprescindible realizar una adecuada retroalimentación. Esta revisión debería resaltar lo que está bien, lo que está mal y explicar lo que se debe mejorar. La formación tecnológica docente es un asunto de gran relevancia de constante interés que ha tenido un crecimiento importante (Rodríguez-García \& Martínez, 2018).

Para fortalecer la relación entre lo que el estudiante aprende en la institución educativa y lo que percibe fuera de ella, es 
tener en cuenta la posibilidad de indagar sobre problemáticas que ocurren en la práctica docente. El aprendizaje vivencial, el cual tiene como objetivo que los estudiantes aprendan cuando participan en experiencias de manera activa y de esta manera aplicar lo que aprenden en situaciones nuevas.

Se distinguen dos tipos de retroalimentaciones: focalizadas en autoestima $y$ focalizadas en la tarea. "Sabemos que la retroalimentación impacta en las personas tanto en sus aspectos emocionales como en sus aspectos cognitivos y no es lo mismo decirle algo a alguien de buena manera que decírselo de mala manera" (Anijovich, 2015). En este sentido, la retroalimentación que se trabaja a través de tareas facilita información respecto al rendimiento a lo aprendido, donde el rol docente es verificar si el estudiante presenta dudas, interrogantes, errores y de esta manera ayudarlo. Retroalimentar positivamente aumenta la autoestima, refuerza la confianza y motiva a querer alcanzar los logros propuestos (ver Figura 8).

Figura 9

Principios para una Retroalimentación Efectiva

\section{Motivación}

Asertividad en los contenidos de los mensajes

Presentar sujerencias o propuestas

\section{Figura 8}

Modalidades de Ayuda

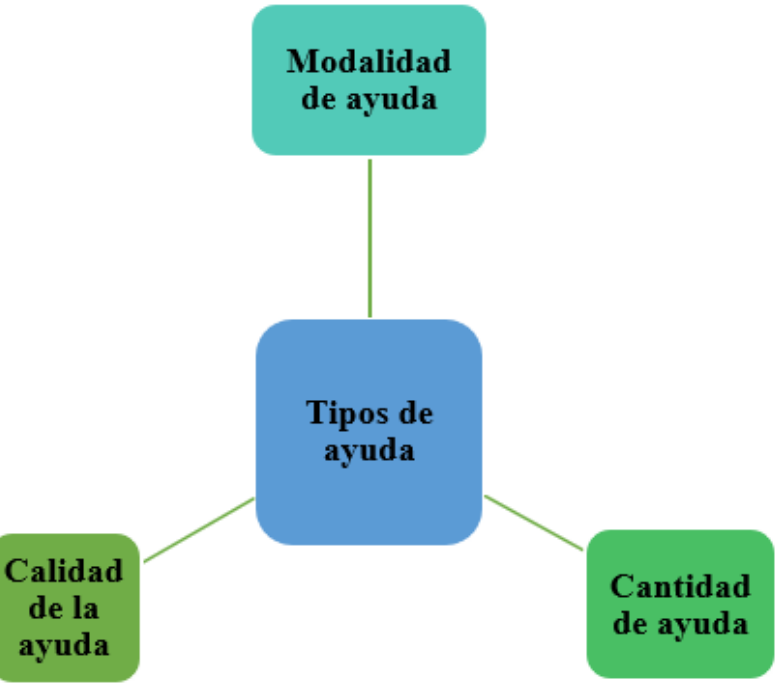

Nota. De acuerdo con la profesora entrevistada los criterios seguidos para los tipos de ayuda se basaron en respetar los estilos personales de los estudiantes, elaboración propia (2021).

El docente debe procura reflexionar sobre estas situaciones de ayuda para poder intervenir desde el conocimiento, comprometiéndose por el estado de la situación de sus estudiantes, y adquiriendo un comportamiento crítico y reflexivo, volviéndose un elemento fundamental para la educación en los tiempos actuales. En este contexto, se han identificado principios básicos para realizar una retroalimentación que se presenta en la Figura 9.

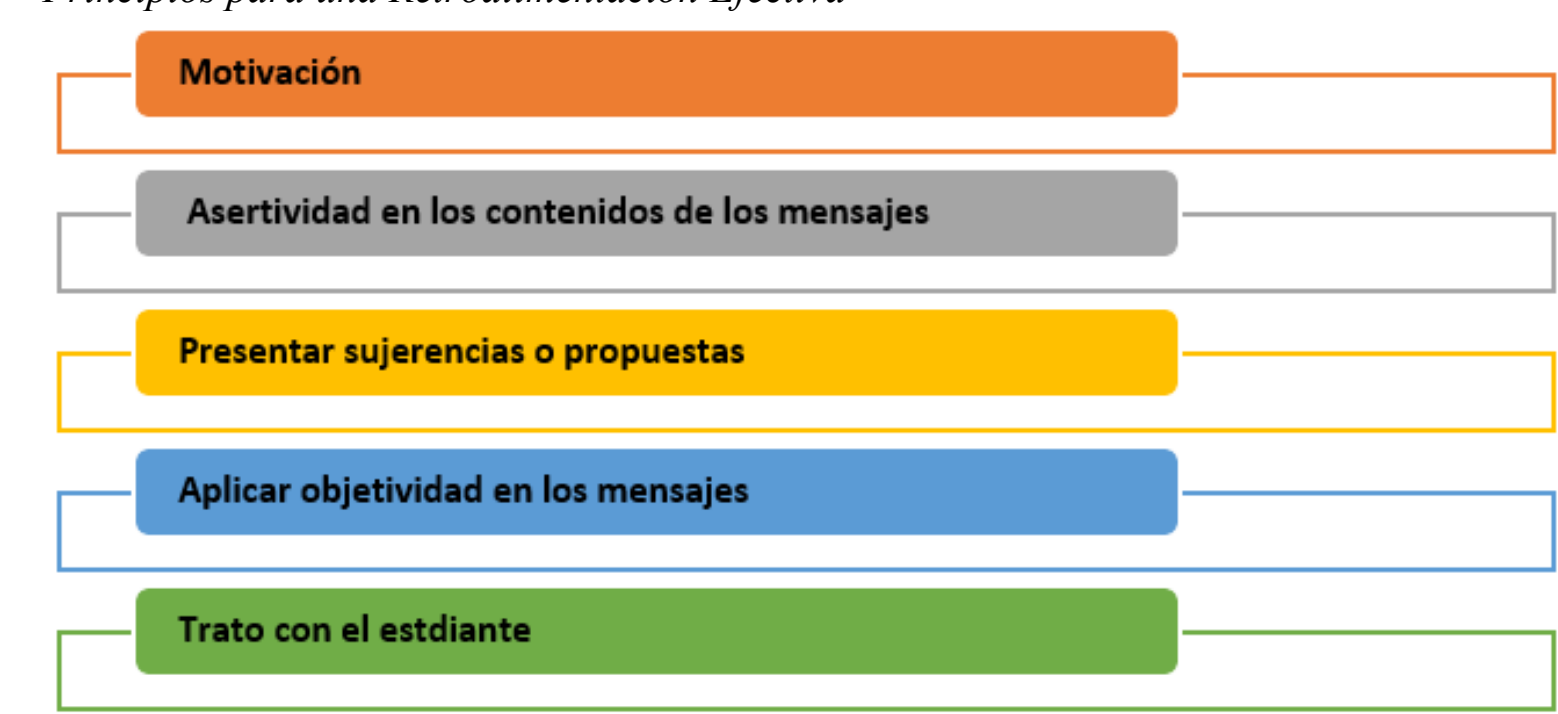

Nota. Principios para una retroalimentación efectiva, elaborado por Ross (2014). 
Los principios antes mencionados, requiere un docente que guie al estudiante a comprender su manera de aprender $\mathrm{y}$ autorregular. El proceso de retroalimentación ayudó al estudiante en la construcción de autonomía a través de la reflexión crítica replanteando sus trabajos, e identificando sus estrategias de aprendizaje de esta manera lograr una autoevaluación de qué aprende y cómo aprende. La retroalimentación efectiva provoca cambios en el estudiante. Si bien la profesora de la materia utilizó los elementos básicos de la retroalimentación es importante para lograr un verdadero proceso de mejora que esta herramienta sirva también para evaluar el propio proceso del docente.

\section{Conclusiones}

Debido a las transformaciones actuales, fue preciso aprender a vivir con incertidumbre. Se necesita desarrollar en ambientes de aprendizaje autonomía en estudiantes y docentes, orientándolos hacia el aprender a aprender, es decir poseer capacidad de reflexionar, analizar, tomar conciencia del saber y estar dispuestos al cambio.

El éxito del nuevo modelo educativo paso en primera instancia por el cambio de paradigma de enseñanza y aprendizaje, dejando de entender al proceso como transmisión de conocimiento. Innovar en educación no pasa solamente por integrar las tecnologías, sino en transformar las prácticas docentes. Es así, que el estudio realizado permitió reflexionar sobre la combinación de necesidades, actitudes, capacitación docente, emociones en la virtualidad, integración del currículo, disposición de recursos tecnológicos y humanos.

Por otro lado, fue necesario un análisis profundo sobre la enseñanza en contextos virtuales como ambiente problematizador, lo cual implica una actitud positiva ante el saber, convirtiendo este fenómeno en objeto de estudio. Resulto pertinente indagar para comprender el papel que juega lo emocional y lo intercultural en contextos virtuales. Esto nos lleva a un análisis profundo de valores insertos en diferentes contenidos, recursos, estrategias y actividades para el aprendizaje. Otro elemento para considerar en el perfil del egresado que, más allá de las competencias académicas es necesario integrar habilidades tecnologías en la formación previa y en la formación permanente.

El currículo en la práctica docente es una herramienta que actúa como dispositivo mediador, basándose no solo en lo que se debe enseñar sino también ayudar a crear espacios de aprendizajes flexibles. Durante las trayectorias educativas de los estudiantes se debe incorporar competencias tecnológicas relacionadas con una formación didáctica emergente y problematizadora.

Como cierre y propuesta de valor se recomiendo que frente a los nuevos modelos de aprendizaje es necesario realizar estudios sobre la una correspondencia entre el perfil del egresado del Nivel Superior, el diseño de ambientes virtuales de aprendizaje y las planificaciones didácticas. Es importante conocer las creencias de los estudiantes para saber si el uso de algo nuevo como el aula virtual se adapta a ellos, ya que, a veces su utilidad no resulta pertinente al contexto, es por ello, que se recomienda investigar sobre este tema.

\section{Referencias}

Anijovich, R. (2015). El valor formativo de la retroalimentación. https://www.youtube. com/watch? $\mathrm{v}=$ ShlEPX6_NUM.

Barron, B. (2006). Interest and self-sustained learning as catalysts of development: A learning ecology perspective. Human development, (4) 193-224. Coaten, N. (2003). Blended e-learning. Educaweb.

Coll, C. (1985). Acción, interacción y construcción del conocimiento en situaciones educativas. Anuario de Psicología, $\mathrm{n}^{\circ} 3$.

Marchesi, A. (2009). Metas educativas 2021. Los desafios de las TIC para el cambio educativo. Santillana

Mujica-Sequera, R. (2020). E-Learning como estrategia pedagógica en la educación superior. Revista Tecnológica-Educativa Docentes 2.0, 9(1), 37-41. https://doi.org/10.37843/rted.v9i1.103

Muñoz, M., Fragueiro, M. S., \& Ayuso, M. J. (2016). La importancia de las redes sociales en el ámbito educativo. Escuela abierta, 16, 91-104. http://www.ceuandalucia.es/escuelaabierta/pdf/artic ulos_ea16_pdf/redes\%2091-104.pdf 
Prensky, M. (2010) Nativos e inmigrantes digitales. SEK 2.0 Editorial. www.marcprensky.com/

Popp, W. (1976). Kommunikative Didaktik. Weinheim. Rockwell, E. (1986a). Cómo observar la reproducción. Revista Colombiana de Educación, 17, 109-125.

Fernández, A. (1992). Roles y funciones en el desarrollo organizacional. Congreso interuniversitario de Organización Escolar. Sevilla

Rodríguez-García, A. M. \& Martínez, N. (2018). La competencia digital en la base de Scopus: un estudio de metaanálisis. REXE-Revista de Estudios y Experiencias en Educación, 2(2), 15-24. http://www.rexe.cl/ojournal/index.php/rexe/article/v iew $/ 544 / 437$

Sánchez-Camacho, J.M. (2019). Estrategias didácticas con el uso de las TIC para el desarrollo de habilidades motoras básicas de los estudiantes de la Institución Educativa Casilda Zafra de Santa Rosa de Viterbo. Tesis de postgrado. Universidad Santo Tomas. Tunja.

Ross, P. \& Tronson, D. (2005). Providing quality feedbackWhere to from here? UniServe Science Blended Learning Symposium Proceedings. https://bit.ly/2sC5INn

Valenzuela, R. (2013). Las redes sociales y su aplicación en la educación. Revista digital universitaria, 14(4). http://www.revista.unam.mx/vol.14/num4/art36/ind ex.html 